\title{
Mobility-Based CAC Algorithm for Arbitrary Call-Arrival Rates in CDMA Cellular Systems
}

\author{
Robert G. Akl, Member, IEEE, Manju V. Hegde, Member, IEEE, and Mort Naraghi-Pour, Member, IEEE
}

\begin{abstract}
This paper presents a novel approach for designing a call-admission control (CAC) algorithm for code-division multiple-access (CDMA) networks with arbitrary call-arrival rates. The design of the CAC algorithm uses global information; it incorporates the call-arrival rates and the user mobilities across the network and guarantees the users' quality of service $(\mathrm{QoS})$ as well as prespecified blocking probabilities. On the other hand, its implementation in each cell uses local information; it only requires the number of calls currently active in that cell. We present several cases for a nontrivial network topology where our CAC algorithm guarantees QoS and blocking probabilities while achieving significantly higher throughput than that achieved by traditional techniques. We also calculate the network capacity, i.e., the maximum throughput for the entire network, for prespecified blocking probabilities and QoS requirements.
\end{abstract}

Index Terms-Call-admission control (CAC) algorithm, code-division multiple-access (CDMA), implied costs, mobility.

\section{INTRODUCTION}

C ALL-ADMISSION control (CAC) algorithms are used to decide whether or not a network should accept a new call. Such algorithms must be designed to guarantee a grade of service (GoS), i.e., the call-blocking rate, as well as a quality of service (QoS), i.e., the probability of the loss of communication quality. A new call is accepted if the network can provide the QoS requested by the call without adversely affecting the QoS of the calls that are currently being carried. Otherwise, the call is rejected. The choice of CAC affects the utilization of network resources and its role is to protect the network and user in order to achieve network-performance objectives.

In code-division multiple-access (CDMA) cellular systems, designing a CAC algorithm for arbitrary call-arrival rates while meeting the above objectives is difficult. Due to self interference in CDMA, the number of simultaneous calls that can be handled within one cell depends on the number of calls being carried in all its neighboring cells. One approach to simplifying the design is to assume equal call-arrival rates with an equal number of simultaneous calls in every cell. In this case, the maximum number of calls $N$ per cell that can be supported while maintaining an acceptable signal-to-interference ratio (SIR) is determined and a possible CAC algorithm would be to allow no more

Manuscript received October 13, 2003; revised March 2, 2004, May 25, 2004, September 28, 2004, and October 5, 2004. The review of this paper was coordinated by Prof. V. Leung.

R. Akl is with the Department of Computer Science and Engineering, University of North Texas, Denton, TX 76207 USA (e-mail: rakl@cse.unt.edu).

M. Hegde is with Ageia Technologies, St. Louis, MO 63108 USA.

M. Naraghi-Pour is with the Department of Electrical and Computer Engineering, Louisiana State University, Baton Rouge, LA 70803 USA.

Digital Object Identifier 10.1109/TVT.2004.841559 than $N$ calls in any one cell; a call arriving at a cell that has $N$ calls in progress is blocked. This is clearly far from optimal as more calls may be accepted in a cell if its neighboring cells carry only a few calls at the time.

Another approach models each cell as an independent $M / M / \infty$ queue [1], implying that no arriving call will be blocked. In [1], the authors emphasize that the notion of blocking used is that of "soft blocking," i.e., when the SIR requirements of all users cannot be met. This model appears in several papers [2]-[4] and is used to study the teletraffic capacity of CDMA cellular networks, but provides no input to the understanding of how calls should be admitted to the system. In [5], a signal-to-interference-based CAC algorithm is proposed based on residual capacity, which the authors define as the additional number of initial calls a base station can accept such that system wide outage probability will be guaranteed to remain below a certain level. In [6], a CAC algorithm is proposed to administer requests based on the required SNR and QoS. In [7], interference-level-based CAC and number-of-users-based CAC are compared and design methods are proposed using expressions for the CAC threshold and the Erlang capacity of the reverse link. Signal-to-interference-based CAC algorithms also appear in [8] by predicting the additional intercell interference the new call will produce, in [9] by using different threshold values in two layer hierarchical cell structure, and in [10] by using upper bounds on the admission threshold. In [11], a method to estimate the set of candidate cells into which a mobile may move in the near future is proposed based on channel reservation. Resource-reservation based CAC algorithms also appear in [12], where a small portion of system resources is reserved for new calls occurring in neighboring cells instead of fully allowing the local users in a home cell to occupy the resources. In [13], an intelligent CAC policy is proposed that can adjust the CAC algorithm and its threshold according to the current traffic load in local and neighboring cells.

Assuming equal call-arrival rates simplifies design but sacrifices performance when, as in most practical cases, traffic is not uniform. On the other hand, designing an optimal CAC algorithm (optimal in the sense that it minimizes the blocking probabilities) for an arbitrary call-arrival rate profile results in a design computational complexity that is exponential in the number of cells [14]. In addition, the CAC algorithm requires global state, i.e., the number of calls in progress in all the cells of the network.

In order to simplify the design of the CAC algorithm, we consider those algorithms that only require local state, i.e., the number of calls in progress in the current cell. We reduce the set of feasible states that a CDMA network can be in to a set of admissible states when calculating the blocking probabilities. 
As a result, the complexity of the calculation of the blocking probabilities becomes linear in the number of cells instead of exponential.

Several mobility models have appeared in the literature [15]-[24]. In our mobility model, a call stops occupying a cell either because user mobility has forced the user to hand the call off to another cell or because the call is completed. To study the effect of mobility, we define a revenue function that is a generalization of the throughput in the network. This revenue consists of two components: the first is the revenue generated by accepting a new call in each cell and the second is the cost of a forced termination due to handoff failure of those calls that have been accepted in a cell. Our CAC algorithm does not distinguish between new and handoff calls when making call-admission decisions. Since handoff failure depends on the CAC used, the revenue depends implicitly on the CAC algorithm. We calculate the implied costs, which are the derivatives of the implicitly defined revenue function, and capture the effect of increases in the maximum number of calls allowed to be admitted in one cell on the revenue of the entire network. A constrained optimization problem is then formulated in order to maximize the revenue subject to upper bounds on the blocking probabilities and a lower bound on the bit-energy-to-interference ratio. The implied costs are used to solve this problem. We present examples of CDMA networks with arbitrary call-arrival rates demonstrating that our $\mathrm{CAC}$ algorithm can guarantee the QoS and achieve lower call-blocking probabilities and higher throughput than traditional CAC algorithms.

The remainder of this paper is organized as follows. Our traffic and mobility models are presented in Section II. We construct our call-admission-control algorithm in Section III and we optimize the throughput and the revenue in Section IV. Numerical results are presented in Section $\mathrm{V}$ and, finally, Section VI concludes this paper. The derivation of the implied costs are given in the Appendix.

\section{TRAFFIC AND MOBILITY MODELS}

\section{A. Feasible States}

Consider a multicell CDMA network with spread signal bandwidth of $W$, information rate of $R$ bits/s, voice activity factor of $\alpha$, and background noise spectral density of $N_{0}$. Assuming a total of $M$ cells with $n_{i}$ calls in cell $i$, the bit-energy-to-interference density ratio in cell $i$ is given by [14], [25]

$$
\left(\frac{E_{b}}{I_{0}}\right)_{i}=\frac{E_{b}}{\frac{\alpha\left(R E_{b}\right)\left(n_{i}-1+\sum_{j=1}^{M} n_{j} \kappa_{j i}\right)}{W}+N_{0}} \text {, for } i=1, \ldots, M \text {, }
$$

where $\kappa_{j i}=I_{j i} / n_{j}$ is the per-user intercell interference factor from cell $j$ to cell $i$ and where $I_{j i}$ denotes the relative average interference of cell $j$ to cell $i$. To achieve a required bit-error rate (BER), we must have $\left(E_{b} / I_{0}\right)_{i} \geq \Gamma$ for some constant $\Gamma$. Thus, rewriting (1), the number of calls in each cell must satisfy

$$
n_{i}+\sum_{j=1}^{M} n_{j} \kappa_{j i} \leq \frac{\frac{W}{R}}{\alpha}\left(\frac{1}{\Gamma}-\frac{1}{\frac{E_{b}}{N_{0}}}\right)+1 \triangleq c_{\mathrm{eff}}, \text { for } i=1, \ldots, M
$$

A set of calls $\mathbf{n}=\left(n_{1}, \ldots, n_{M}\right)$ satisfying the previous equations is said to be a feasible call configuration or a feasible state, i.e., one that satisfies the $\left(E_{b} / I_{0}\right)$ constraint. The right-hand side of (2) is a constant that is determined by system parameters and by the desired maximum BER and can be regarded as the total number of effective channels $c_{\text {eff }}$ available to the system.

Denote by $\Omega$ the set of feasible states. Define the set of blocking states for cell $i$ as

$$
\mathcal{B}_{i}=\left\{\mathbf{n} \in \Omega:\left(n_{1}, \ldots, n_{i}+1, \ldots, n_{M}\right) \notin \Omega\right\}
$$

If a new call or a handoff call arrives to cell $i$, it is blocked if the current state of the network $\mathbf{n}$ is in $\mathcal{B}_{i}$.

\section{B. Mobility Model}

The call-arrival process to cell $i$ is assumed to be a Poisson process with rate $\lambda_{i}$ independent of other call-arrival processes. In the case of equal call-arrival rates, $\lambda_{i}=\lambda$ for all $i$. The call dwell time is a random variable with exponential distribution having mean $1 / \mu$ and is independent of earlier arrival times, call durations, and elapsed times of other users. At the end of a dwell time, a call may stay in the same cell, attempt a handoff to an adjacent cell, or leave the network. Define $q_{i i}$ as the probability that a call in progress in cell $i$ remains in cell $i$ after completing its dwell time. In this case, a new dwell time that is independent of the previous dwell time begins immediately. Let $q_{i j}$ be the probability that a call in progress in cell $i$ after completing its dwell time goes to cell $j$. If cells $i$ and $j$ are not adjacent, then $q_{i j}=0$. We denote by $q_{i}$ the probability that a call in progress in cell $i$ departs from the network.

This mobility model is attractive because we can easily define different mobility scenarios by varying the values of these probability parameters [26]. For example, if $q_{i}$ is constant for all $i$, then the average dwell time of a call in the network will be constant regardless of where the call originates and what the values of $q_{i i}$ and $q_{i j}$ are. Thus, in this case, by varying $q_{i i}$ 's and $q_{i j}$ 's, we can obtain low- and high-mobility scenarios and compare the effect of mobility on network attributes (e.g., throughput).

We assume that the occupancy of the cells evolves according to an $M$-dimensional birth-death process, where the total arrival rate or offered traffic to cell $i$ is $\rho_{i}$ and the departure rate from cell $i$ when the network is in state $\mathbf{n}$ is $n_{i} \mu_{i}=n_{i} \mu\left(1-q_{i i}\right)$. Let $\underline{\rho}$ be the vector of offered traffic to the cells, let $\underline{\mu}$ be the vector of

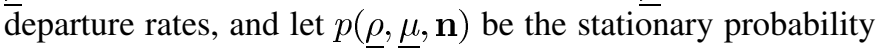
that the network is in state $\mathbf{n}$. The distribution $p$ is obtained as

$$
p(\underline{\rho}, \underline{\mu}, \mathbf{n})= \begin{cases}P_{0} \prod_{k=1}^{M} \frac{\left(\frac{\rho_{k}}{\mu_{k}}\right)^{n_{k}}}{n_{k} !}, & n_{i}+\sum_{j=1}^{M} n_{j} \kappa_{j i} \leq c_{\mathrm{eff}} \\ 0, & \text { for } i=1, \ldots, M \\ & \text { otherwise }\end{cases}
$$

where $P_{0}$ is a normalizing constant such that $\sum_{\mathbf{n} \in \Omega} p(\underline{\rho}, \underline{\mu}, \mathbf{n})=1$. The new call-blocking probability for cell $i, B_{i}$, is given by

$$
B_{i}=\sum_{\mathbf{n} \in \mathcal{B}_{i}} p(\underline{\rho}, \underline{\mu}, \mathbf{n})
$$


This also is the blocking probability of handoff calls due to the fact that handoff and new calls are treated in the same way by the network.

Let $\mathcal{A}_{i}$ be the set of cells adjacent to cell $i$. Let $\nu_{j i}$ be the handoff rate out of cell $j$ offered to cell $i . \nu_{j i}$ is the sum of the proportion of new calls accepted in cell $j$ that go to cell $i$ and the proportion of handoff calls accepted from cells adjacent to cell $j$ that go to cell $i$. Thus

$$
\nu_{j i}=\lambda_{j}\left(1-B_{j}\right) q_{j i}+\left(1-B_{j}\right) q_{j i} \sum_{x \in \mathcal{A}_{j}} \nu_{x j}
$$

which can be rewritten as

$$
\nu_{j i}=\nu\left(B_{j}, \rho_{j}, q_{j i}\right)=\left(1-B_{j}\right) q_{j i} \rho_{j}
$$

where $\rho_{j}$, the total offered traffic to cell $j$, is given by

$$
\rho_{j}=\rho\left(\mathbf{v}, \lambda_{j}, \mathcal{A}_{j}\right)=\lambda_{j}+\sum_{x \in \mathcal{A}_{j}} \nu_{x j}
$$

and where $\mathbf{v}$ denotes the matrix whose components are the handoff rates $\nu_{i j}$ for $i, j=1, \ldots M$.

The total offered traffic can be obtained from a fixed point model [27], which describes the offered traffic as a function of the handoff and new call-arrival rates, the handoff rates as a function of the blocking probabilities and the offered traffic and the blocking probabilities as a function of the offered traffic. For a given set of arrival rates, we use an iterative method to solve the fixed point equations and define an initial value for the handoff rates. We calculate the offered traffic by adding the given values of the arrival rates to the handoff rates. The blocking probabilities are now calculated using the offered traffic. We then calculate the new values of the handoff rates and repeat. This approach has been extensively utilized in the literature to obtain solutions of fixed point problems [28]-[33]. The questions of the existence and uniqueness of the solution and whether the iterative approach in fact converges to the solution (if a unique solution exists) are generally difficult to answer due to the complexity of the equations involved. Kelly has shown that, for fixed alternate routing, the solution to the fixed point problem is in fact not unique [34]; in all the numerical examples we solved, the iterative approach converged to a unique solution.

\section{CAC ALGORITHM}

A CAC algorithm can be constructed as follows. A call arriving to cell $i$ is accepted if and only if the new state is a feasible state. Clearly, this CAC algorithm requires global state, i.e., the number of calls in progress in all the cells of the network. Furthermore, to compute the blocking probabilities, the probability of each state in the feasible region needs to be calculated. Since the cardinality of $\Omega$ is $O\left(c_{\mathrm{eff}}^{M}\right)$, the calculation of the blocking probabilities has a computational complexity that is exponential in the number of cells.

In order to simplify the CAC algorithm, we consider only those $\mathrm{CAC}$ algorithms that utilize local state, i.e., the number of calls in progress in the current cell. To this end, we define a state $\mathbf{n}$ to be admissible if

$$
n_{i} \leq N_{i} \quad \text { for } \quad i=1, \ldots, M
$$

where $N_{i}$ is a parameter that denotes the maximum number of calls allowed to be admitted in cell $i$. Clearly, the set of admissible states denoted $\Omega^{\prime}$ is a subset of the set of feasible states $\Omega$. The blocking probability for cell $i$ is then given by

$$
B_{i}=B\left(A_{i}, N_{i}\right)=\frac{\frac{A_{i}^{N_{i}}}{N_{i} !}}{\sum_{k=0}^{N_{i}} A_{i}^{k} / k !}
$$

where $A_{i}=\rho_{i} / \mu_{i}=\rho_{i} / \mu\left(1-q_{i i}\right)$ is the Erlang traffic in cell $i$. We note that the complexity to calculate the blocking probabilities in (10) is $O(M)$ and the BER requirement is guaranteed since $\Omega^{\prime} \subset \Omega$.

Once the maximum number of calls that are allowed to be admitted in each cell $\left(N_{1}, \ldots, N_{M}\right)$ is calculated (this is done offline and described in the next section), the CAC algorithm for cell $i$ will simply compare the number of calls currently active in cell $i$ to $N_{i}$ in order to accept or reject a new arriving call. Thus, our CAC algorithm has a computational complexity that is $O(1)$.

\section{ThroughPUT AND NETWORK CAPACITY}

The throughput of cell $i$ consists of two components: the new calls that are accepted in cell $i$ minus the forced termination due to handoff failure of the handoff calls into cell. Hence, the total throughput $T$ of the network is

$$
T(\mathbf{B}, \underline{\rho}, \underline{\lambda})=\sum_{i=1}^{M}\left\{\lambda_{i}\left(1-B_{i}\right)-B_{i}\left(\rho_{i}-\lambda_{i}\right)\right\}
$$

where $\mathbf{B}$ is the vector of blocking probabilities and $\underline{\lambda}$ is the vector of call-arrival rates.

To study the effect of mobility and to differentiate between new and handoff calls, the throughput function can be generalized to a revenue function. The term revenue, suggesting an economic meaning, is chosen to emphasize the rewards from not blocking a new call and the penalty (whether measured monetarily or by customer aggravation) from having handoff calls blocked. Hence, the revenue $H$ becomes

$$
H(\mathbf{B}, \underline{\rho}, \underline{\lambda})=\sum_{i=1}^{M}\left\{w_{i} \lambda_{i}\left(1-B_{i}\right)-c_{i} B_{i}\left(\rho_{i}-\lambda_{i}\right)\right\}
$$

where $w_{i}$ is the revenue generated by accepting a new call in cell $i$ and $c_{i}$ is the cost of a forced termination of a call due to a handoff failure in cell $i$. The values of $w_{i}$ and $c_{i}$ control the tradeoff between new and handoff calls. The choice of $w_{i}$ and $c_{i}$ affect the CAC algorithm through their effect on $\left(N_{1}, \ldots, N_{M}\right)$. For example, assume that $w_{i}=1$ and $c_{i}=c$ for all $i$. Then, it is easy to see that for a given network topology the choice of larger $c$ will tend to increase the values of $N_{i}$ for those cells into which the handoff rates are high while decreasing the values of 
$N_{i}$ for the other cells. These are tools that allow the system administrator to place more importance on handoff calls. Surveys and market studies investigating these issues help network administrators to set these weights and achieve a desired tradeoff.

\section{A. Calculation of $\left(N_{1}, \ldots, N_{m}\right)$}

We formulate a constrained optimization problem in order to maximize the revenue subject to upper bounds on the blocking probabilities and a lower bound on the signal-to-interference constraints in (2). The goal is to optimize the utilization of network resources and provide consistent GoS while at the same time maintaining the QoS for all the users. In this optimization problem, the arrival rates are given and the maximum number of calls that can be admitted in all the cells are the independent variables. This is given in

$$
\begin{array}{cl}
\max _{\left(N_{1}, \ldots, N_{M}\right)} & H(\mathbf{B}, \underline{\rho}, \underline{\lambda}) \\
\text { subject to } & B\left(A_{i}, N_{i}\right) \leq \eta \\
& N_{i}+\sum_{j=1}^{M} N_{j} \kappa_{j i} \leq c_{\mathrm{eff}} \\
& \text { for } \quad i=1, \ldots, M .
\end{array}
$$

The optimization problem in (13) is solved offline to obtain the values of $\left(N_{1}, \ldots, N_{M}\right)$.

In a network's coverage area, the call-arrival rate profile $\left(\lambda_{1}, \ldots, \lambda_{M}\right)$ will change from time to time. By solving the optimization problem in (13), one can compute the values of $\left(N_{1}, \ldots, N_{M}\right)$ for different call-arrival rate profiles and store these in the mobile switching center along with the time periods of the corresponding profiles. A dynamic CAC algorithm can then be implemented whereby the optimized values of $\left(N_{1}, \ldots, N_{M}\right)$ are used during each corresponding time period. This is reminiscent of the dynamic nonhierarchical routing that was introduced in the mid 1980s in the long-distance AT\&T network [35]. This approach for designing the CAC also allows different thresholds for blocking to be set in individual cells.

In the following, we compare our results to a CAC algorithm where the maximum number of calls that can be admitted in each cell is the same, i.e., $N_{1}=N_{2}=\ldots=N_{M}=N$ (irrespective of the call-arrival rate profile in the network). We also optimize the revenue for this algorithm subject to a lower bound on the bit-energy-to-interference ratio. The problem is formulated as

$$
\begin{aligned}
\max _{(N)} & H(\mathbf{B}, \underline{\rho}, \underline{\lambda}) \\
\text { subject to } & N+\sum_{j=1}^{M} N \kappa_{j i} \leq c_{\mathrm{eff}} \\
& \text { for } \quad i=1, \ldots, M .
\end{aligned}
$$

In the sequel, this optimized algorithm is referred to as the $o p$ timized traditional CAC algorithm. This algorithm will be optimal in the sense of (13) in the case of equal call-arrival rates, equal mobility probabilities for all the cells, and a network with a large number of cells (in which edge effects can be ignored). In this formulation, maximizing the revenue results in the largest possible $N$ that satisfies the signal-to-interference requirements.
This, in turn, results in the smallest possible blocking probabilities for the cells. Thus, in this optimization, we cannot impose an upper bound on the blocking probabilities and the GoS cannot be guaranteed by the resulting CAC algorithm.

\section{B. Network Capacity}

A third optimization problem can be formulated in which the arrival rates and the maximum number of calls that can be admitted in all the cells are the independent variables and objective function is the throughput. This is given in

$$
\begin{aligned}
\max _{\left(\lambda_{1}, \ldots, \lambda_{M}\right),\left(N_{1}, \ldots, N_{M}\right)} & T(\mathbf{B}, \underline{\rho}, \underline{\lambda}) \\
\text { subject to } & B\left(A_{i}, N_{i}\right) \leq \eta \\
& N_{i}+\sum_{j=1}^{M} N_{j} \kappa_{j i} \leq c_{\text {eff }} \\
& \text { for } \quad i=1, \ldots, M .
\end{aligned}
$$

The optimized objective function of (15) provides an upper bound on the total throughput that the network can carry. This is the network capacity for the given GoS and QoS.

The optimization problems in (13) and (14) are integer programming (IP) problems. The optimization problem in (15) is a mixed IP (MIP) problem. One technique to solve the IP/MIP problem is based on dividing the problem into a number of smaller problems in a method called branch and bound [36]. Branch and bound is a systematic method for implicitly enumerating all possible combinations of the integer variables in a model. In this approach, the number of subproblems and branches required can become extremely large.

By relaxing the integer variables $N_{i}, i=1, \ldots, M$, to continuous variables, the optimizations in (13)-(15) are solved using a sequential quadratic programming (SQP) method [37]. In this method, a quadratic programming subproblem is solved at each iteration. A solution to the fixed point equations is calculated iteratively as discussed in Section II. An estimate of the Hessian of the Lagrangian is updated at each iteration using the Broyden-Fletcher-Goldfarb-Shanno (BFGS) formula [38]. A line search is performed using a merit function [39]. The quadratic programming subproblem is solved using an active set strategy [40].

In order to use the SQP method, we need to evaluate the derivatives of $H$ with respect to $\mathbf{N}$ and $\underline{\lambda} . H(\mathbf{B}, \rho, \underline{\lambda})$ is an implicit function of $\mathbf{N}=\left(N_{1}, \ldots, N_{M}\right)$ and $\underline{\lambda}$. We can obtain relations of total and partial derivatives of the revenue by differentiating the fixed point equations. These relations are manipulated to obtain a system of linear equations in the derivatives of the offered traffic with respect to the number of calls admitted and the arrival rates. This allows us to calculate the implied cost, i.e., the derivative of $H$, with respect to the implicit variable $\mathbf{N}$ and with respect to $\underline{\lambda}$. The calculation of the implied costs are given in the Appendix.

The optimization problems in (13)-(15) are not convex optimization problems, so it may be possible for the approaches described before not to converge to a global optimal solution. In order to ensure that this did not occur, we verified the results of the SQP optimizations for a few select cases by using simulated annealing (SA) [41]. SA is an optimization method 


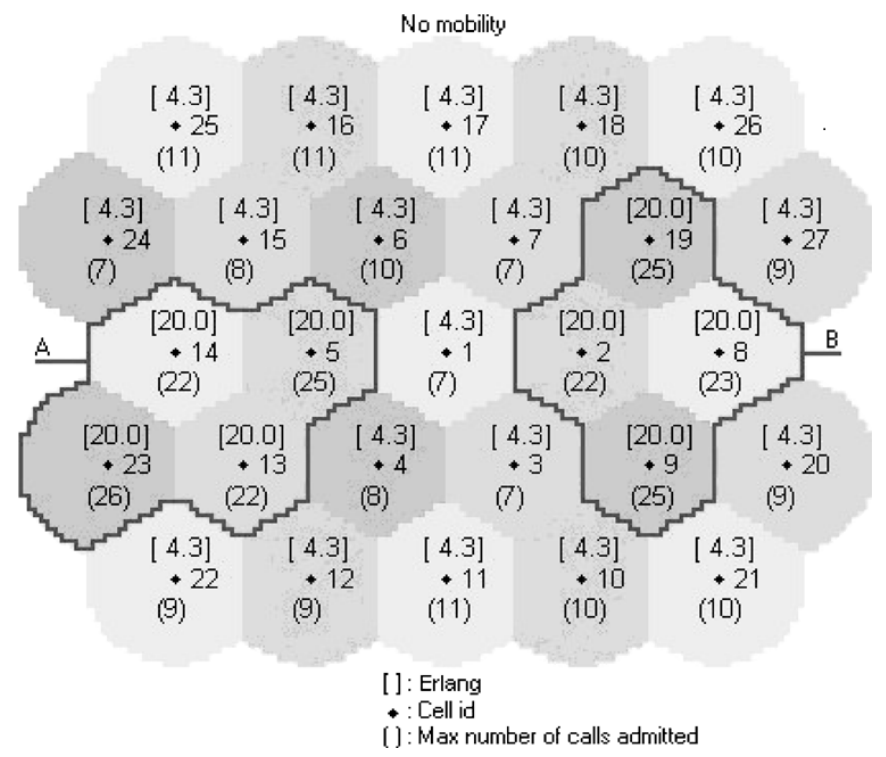

Fig. 1. Erlang traffic and maximum number of calls allowed to be admitted per cell for a network with no mobility of users.

that has many attractive features. In particular, it can statistically guarantee finding a globally optimal solution [42]. However, SA can be quite time consuming to find an optimal solution. Details on our SA parameters and implementation are given in [25]. For computational speed, we have used the SQP optimization method for all the results presented in this paper.

\section{NUMERICAL RESULTS}

The following results have been obtained for the 27-cell CDMA network shown in Fig. 1. The base stations are located at the centers of a hexagonal grid whose radius is $1732 \mathrm{~m}$. Base station 1 is located at the center; the base stations are numbered consecutively in a spiral pattern. The COST-231 propagation model [43] with a carrier frequency of $1800 \mathrm{MHz}$, average base station height of $30 \mathrm{~m}$, and average mobile height of 1.5 $\mathrm{m}$ is used to determine the coverage region. We assume the following for the analysis. The path-loss coefficient is 4 . The shadow fading standard deviation is $6 \mathrm{~dB}$ and the processing gain is $21.1 \mathrm{~dB}$. The bit-energy-to-interference ratio threshold $\Gamma$ is $9.2 \mathrm{~dB}$. The interference-to-background-noise ratio is 10 $\mathrm{dB}$. The voice activity factor is 0.375 . For more details on the choice of these parameters, refer to [44]. Per-user intercell interference factors are evaluated numerically by dividing the whole area into small grids of size $150 \mathrm{~m} \times 150 \mathrm{~m}$ (for more detail, see [25]).

We consider three mobility scenarios: no mobility, low mobility, and high mobility of users. The following probabilities are chosen for the no-mobility case: $q_{i j}=0, q_{i i}=0.3$, and $q_{i}=0.7$ for all cells $i$ and $j$. For the low- and high-mobility case, the mobility probability parameters are given in Tables I and II, respectively. As the mobility of users increases, the total offered traffic in the cells with low exogenous arrival rates also increases due to increased handoff from cells with high arrival rates. We have chosen the parameters of the high-mobility case very high, in order to illustrate that the CAC design algorithms would respond accordingly by assigning more users to the cells
TABLE I

LOW MOBILITY PROBABILITIES

\begin{tabular}{c||c|c|c}
\hline$\left\|\mathcal{A}_{i}\right\|$ & $q_{i j}$ & $q_{i i}$ & $q_{i}$ \\
\hline \hline 3 & 0.020 & 0.240 & 0.700 \\
\hline 4 & 0.015 & 0.240 & 0.700 \\
\hline 5 & 0.012 & 0.240 & 0.700 \\
\hline 6 & 0.010 & 0.240 & 0.700 \\
\hline \hline
\end{tabular}

TABLE II

High MoBILITY PROBABILITIES

\begin{tabular}{c||c|c|c}
\hline$\left\|\mathcal{A}_{i}\right\|$ & $q_{i j}$ & $q_{i i}$ & $q_{i}$ \\
\hline \hline 3 & 0.100 & 0.000 & 0.700 \\
\hline 4 & 0.075 & 0.000 & 0.700 \\
\hline 5 & 0.060 & 0.000 & 0.700 \\
\hline 6 & 0.050 & 0.000 & 0.700 \\
\hline \hline
\end{tabular}

- $\left\|\mathcal{A}_{i}\right\|$ is the number of cells adjacent to cell $i$.

- $q_{i j}$ is the probability a call in cell $i$ goes to cell $j$.

- $q_{i i}$ is the probability a call in cell $i$ stays in cell $i$.

- $q_{i}$ is the probability a call in cell $i$ leaves the network.

with low arrival rates. In all three cases, the probability that a call leaves the network after completing its dwell time is 0.7. Thus, the average dwell time of a call in the network is constant regardless of where the call originates and the mobility scenario used. Consequently, for the same exogenous arrival rates, the Erlang traffic for the entire network is independent of the mobility scenario. This allows us to make meaningful comparisons between different mobility scenarios.

\section{A. Unequal Arrival Rates With the Arrival Rates Given}

In what follows, the revenue generated by accepting a new call in cell $i, w_{i}$ is chosen to be 1 for all the cells. The cost of rejecting a handoff call in cell $i, c_{i}$ is chosen to be 10 for all the cells. We chose $c_{i}$ to be substantially higher to emphasize the priority of the handoff calls. The blocking probability threshold $\eta$ is set to 0.1 .

The call-arrival rates in the network are not equal. This is modeled as two different call-arrival rates. We choose the call-arrival rates to be equal to 14 calls per unit time for all the cells in Group A (i.e., cells 5, 13, 14, and 23) and Group B (i.e., cells 2, 8, 9, and 19), as shown in Fig. 1. For the remaining cells, the call-arrival rates are equal to three calls per unit time. The Erlang traffic per cell (the sum of the call-arrival rates and the handoff rates, i.e., the total offered traffic, divided by the departure rates) is shown in square brackets for the no-, low-, and high-mobility cases in Figs. 1-3, respectively. The maximum number of calls that can be admitted in each cell, calculated from (13), is shown in parentheses in these figures. If the optimized traditional CAC algorithm was to be implemented for the same values of call-arrival rates, system parameters, and the bit-energy-to-interference ratio requirement, the maximum number of calls that could be admitted in each cell would be 18. Such an algorithm sets the threshold of 18 calls per cell for the entire network, irrespective of the actual traffic in each cell. Clearly, this is inefficient in a CDMA network whereby more calls can be admitted in a cell if its neighboring cells have less traffic. In our algorithm for the no-mobility case, it can be seen that for the cells belonging to Groups $\mathrm{A}$ and $\mathrm{B}$, the maximum number of calls admitted has increased from 18 to $22-26$, 


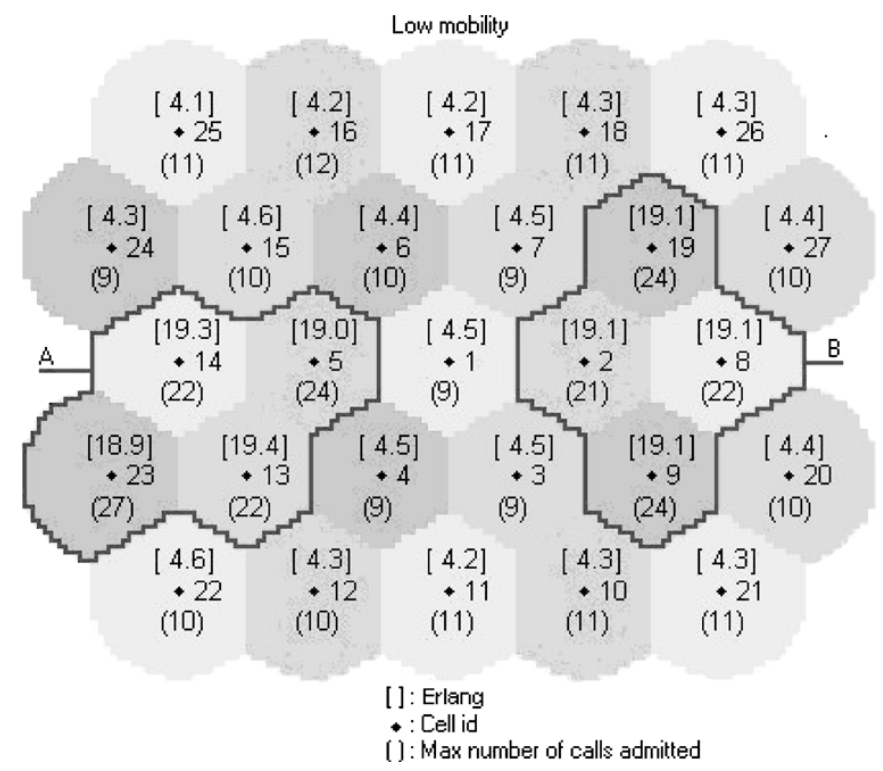

Fig. 2. Erlang traffic and the maximum number of calls allowed to be admitted per cell for a network with low mobility of users.

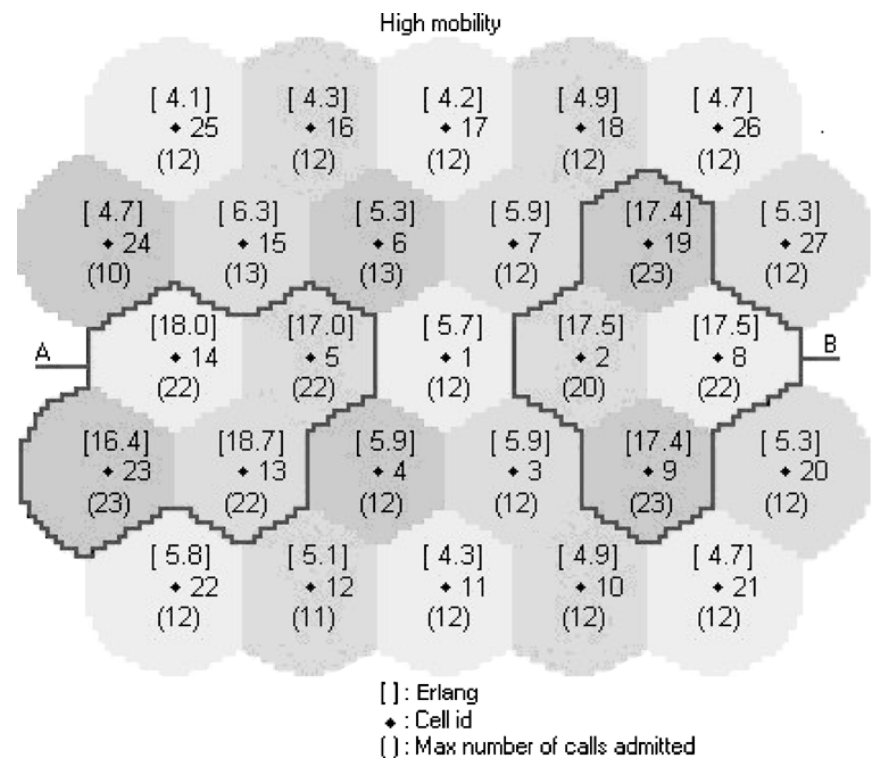

Fig. 3. Erlang traffic and maximum number of calls allowed to be admitted per cell for a network with high mobility of users.

while for all other cells it has decreased from 18 to $7-11$. Our algorithm trades off the calls in the cells with low arrival rate for the calls in the cells with high arrival rate. In what follows, the effect of this will be demonstrated more clearly when we present the blocking probabilities.

As the mobility increases, the handoff rates increase. As a result, the total offered traffic to the cells changes. In particular, the Erlang traffic per cell for cells in groups A and B decreases and, for some of the remaining cells, it increases. Moreover, the Erlang traffic for the cells in a given group is no longer constant, resulting from the fact that the set of neighbors of these cells are different. Note, however, that the total Erlang traffic for the entire network is the same for all three mobility cases.

Figs. 2 and 3 demonstrate that, as the Erlang traffic per cell varies (due to higher mobility and increased handoff), our CAC

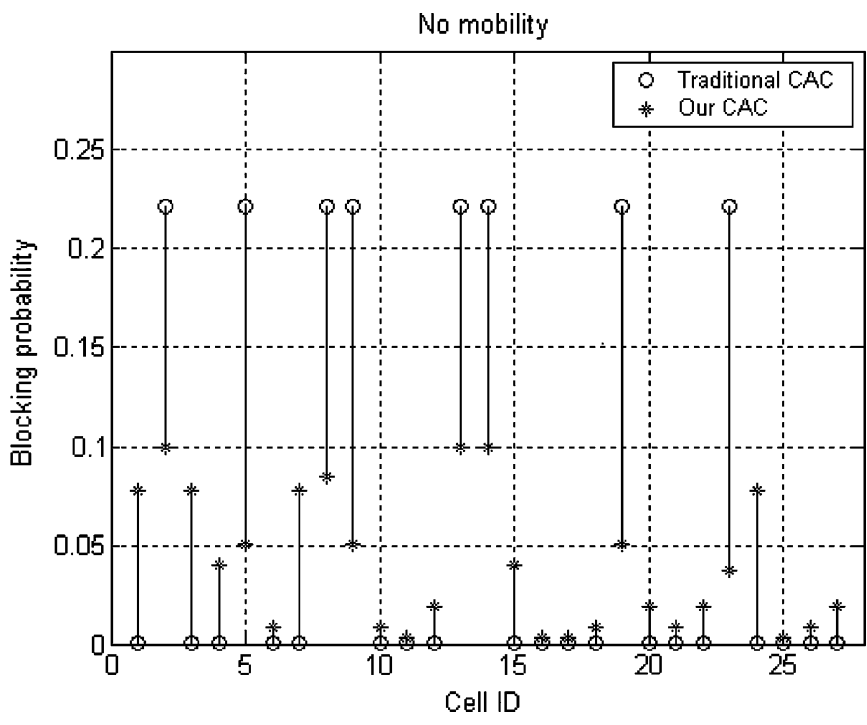

Fig. 4. Blocking probability in each cell for the network in Fig. 1.

algorithm appropriately adjusts the number of calls that can be admitted into each cell. For example, in Fig. 1, the Erlang traffic for cell 1 is 4.3 and the number of calls that can be admitted in this cell is seven. On the other hand, in Fig. 3, the Erlang traffic for this cell has increased to 5.7 and the number of calls that can be admitted has also increased to 12. As another example, in Fig. 1, the Erlang traffic for cell 5 is 20 and the number of calls that can be admitted is 25 . On the other hand, in Fig. 3, the Erlang traffic for this cell has decreased to 17 and the number of calls that can be admitted has also decreased to 22. A similar effect is observed in the other cells in the sense that, to the extent possible, our CAC algorithm adapts the number of calls that can be admitted in each cell in response to the changes in traffic demand to user mobility.

The values of the blocking probabilities per cell resulting from our algorithm and the optimized traditional CAC algorithm for the no-, low-, and high-mobility cases are given in Figs. 4-6, respectively. In these figures, the circle and star at the two ends of a vertical bar indicates the blocking probabilities of the optimized traditional CAC algorithm and our algorithm, respectively, for the cell whose identification number (ID) is shown on the horizontal axis. The optimized traditional CAC algorithm has almost zero blocking probability for cells with low Erlang traffic. On the other hand, the blocking probability for the cells with high Erlang traffic is unacceptably large. This disparity, is of course, undesirable. Our algorithm, on the other hand, achieves a better balance between the blocking probabilities of the low- and high-traffic cells. Due to its call tradeoffs between low- and high-traffic cells, our algorithm is able to accommodate the unequal call-arrival rates of the network and achieve lower blocking probabilities in all the cells. In fact, as the example in Figs. 4-6 demonstrate, our algorithm provides guaranteed GoS (blocking probability less than $\eta$ ) for all the cells in the network for arbitrary call-arrival rates (if a solution exists).

\section{B. Unequal Arrival Rates With the Arrival Rates Not Given}

In what follows, we illustrate the maximization of throughput using (15). The blocking probability threshold $\eta$ is set to 0.02 . 


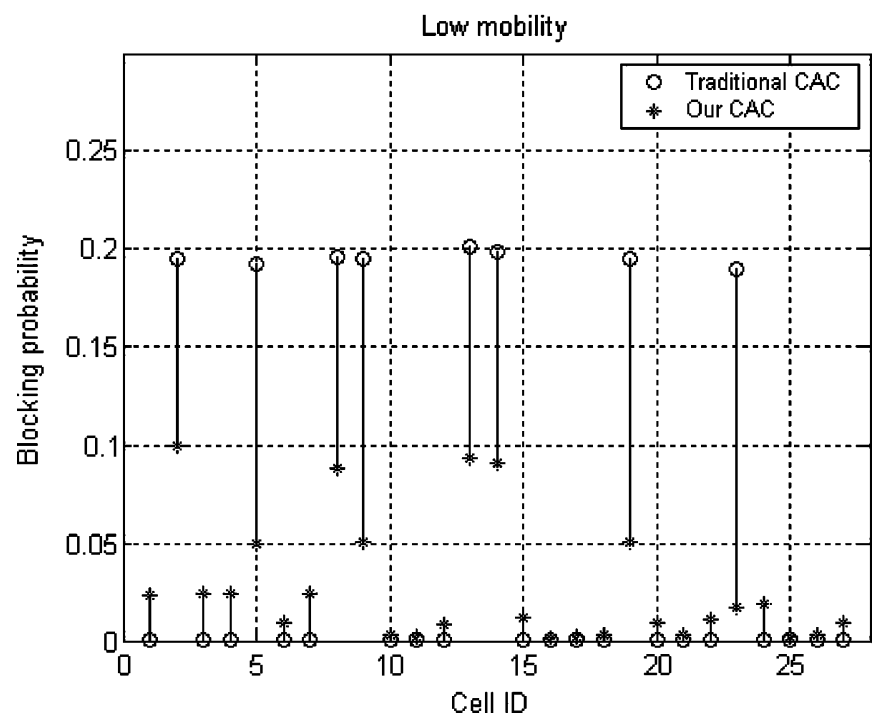

Fig. 5. Blocking probability in each cell for the network in Fig. 2.

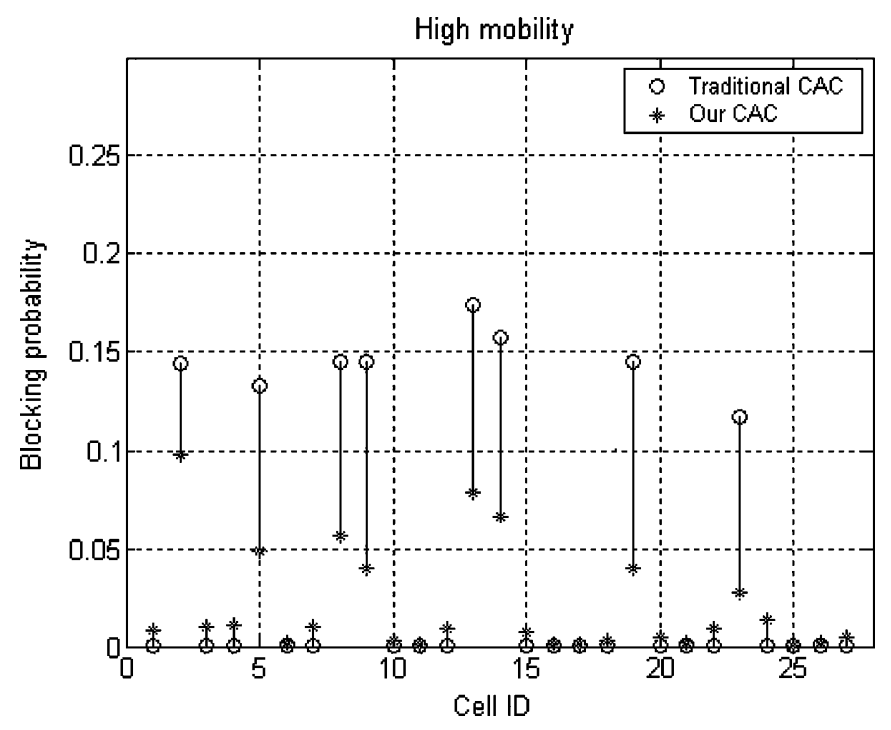

Fig. 6. Blocking probability in each cell for the network in Fig. 3.

We choose the call-arrival rates to be equal to $\lambda$ calls per unit time for all cells except those in group A (i.e., cells 5, 13, 14 , and 23) and group B (i.e., cells 2, 8, 9, and 19), as shown in Fig. 7. For groups A and B, the call-arrival rates are equal to $5 \lambda$ calls per unit time. We solved the optimization given in (15) with the independent variables being $\left(N_{1}, \ldots N_{M}\right)$ and $\lambda$. The total offered traffic per cell (the sum of the call-arrival and handoff rates) is shown in square brackets for the no-, low-, and high-mobility cases in Figs. 7-9, respectively. The maximum number of calls that can be admitted in each cell, calculated from (15), is shown in parentheses in these figures. For the optimized traditional CAC algorithm with the same blocking probability threshold, system parameters, and bit-energy-to-interference ratio requirement, the maximum number of calls that could be admitted in each cell would be 18 . In our algorithm for the no-mobility case, it can be seen that, for the cells belonging to groups $\mathrm{A}$ and $\mathrm{B}$, the maximum number of calls admitted has increased from 18 to $22-24$, while for all other cells it has decreased from 18 to 7-9. Our algorithm trades off the calls in the

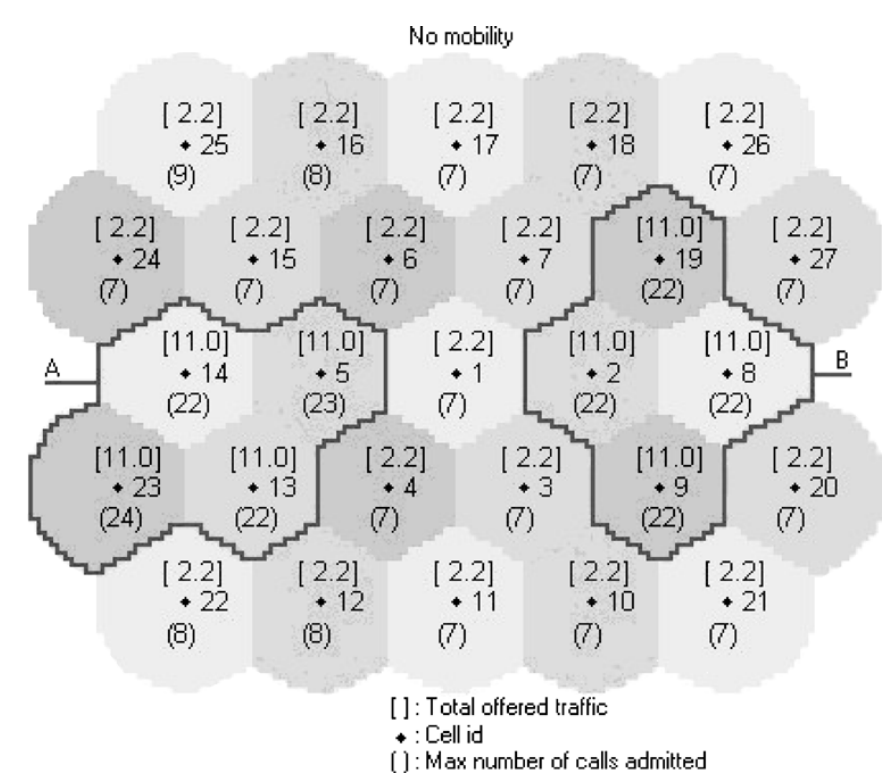

Fig. 7. Total offered traffic and maximum number of calls allowed to be admitted per cell for the 27-cell CDMA network with no mobility of users.

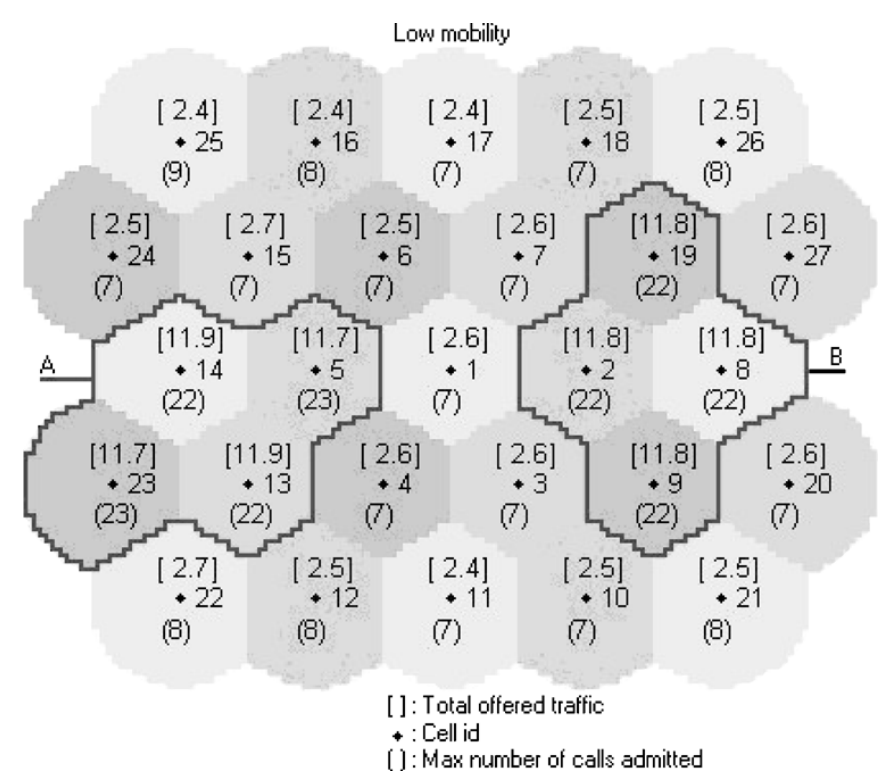

Fig. 8. Total offered traffic and maximum number of calls allowed to be admitted per cell for the 27-cell CDMA network with low mobility of users.

cells with low arrival rate for the calls in the cells with a high arrival rate. As the mobility model changes from no mobility to high mobility, the handoff rates increase, thus increasing the total offered traffic per cell. For the high-mobility case (Fig. 9), the maximum number of calls admitted now ranges from 20 to 23 for cells belonging to groups A and B and from 7 to 11 for all other cells.

The throughput of each cell resulting from our algorithm and the optimized traditional CAC algorithm for the no-, low-, and high-mobility cases are given in Figs. 10-12, respectively. The optimized traditional CAC algorithm has a total network throughput equal to $96.03,99.08$, and 102.43 calls per unit time for the no-, low-, and high-mobility cases, respectively. Our optimization increases the throughput for the network to 127.02, 131.40, and 136.52 calls per unit time for the no-, low-, 


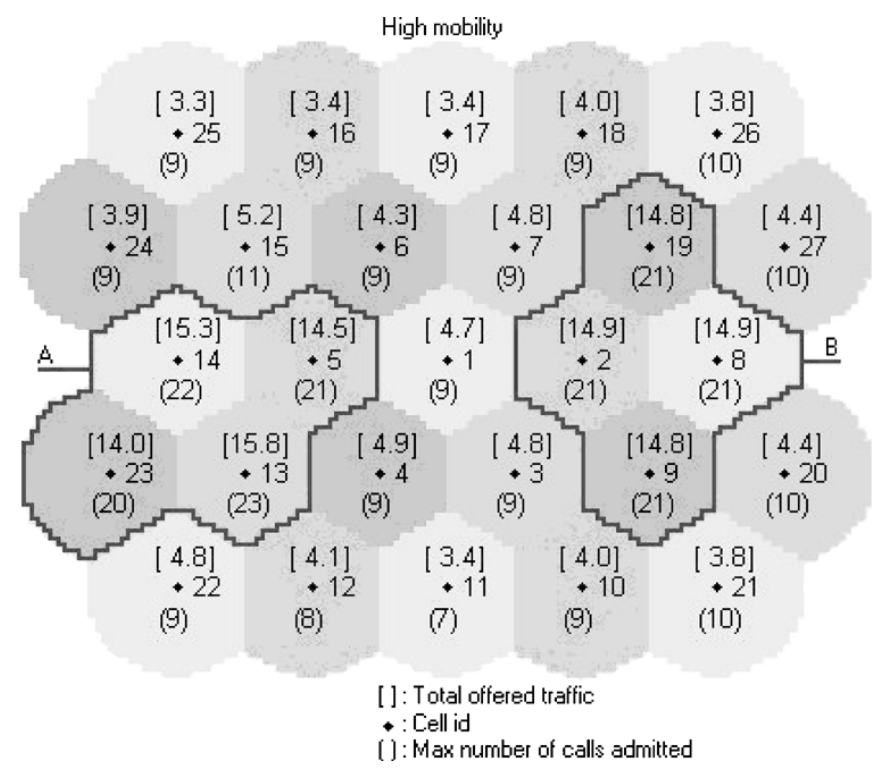

Fig. 9. Total offered traffic and maximum number of calls allowed to be admitted per cell for the 27-cell CDMA network with high mobility of users.

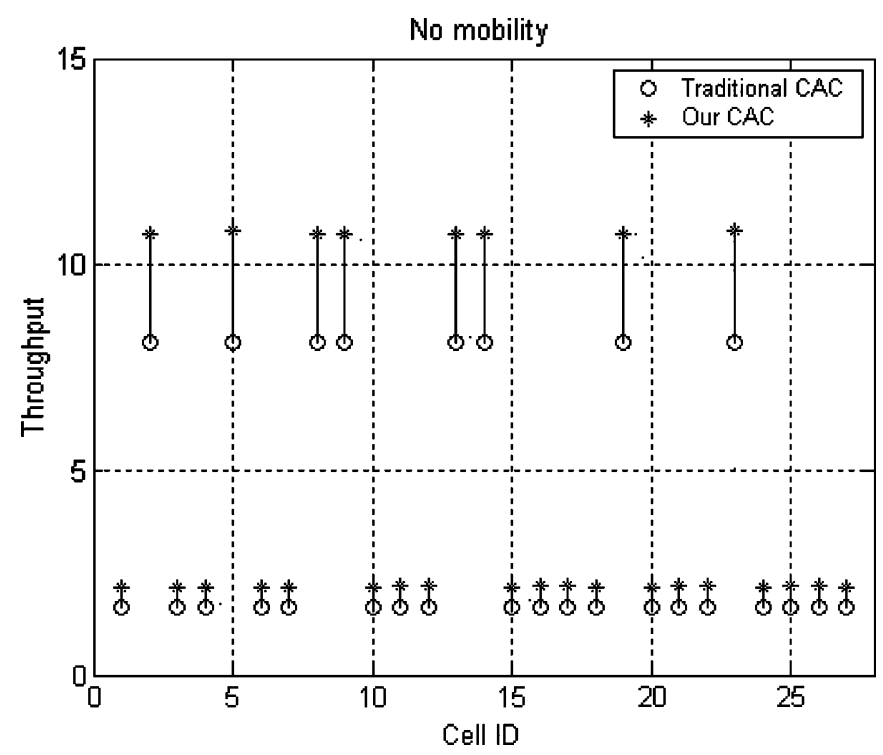

Fig. 10. Maximum throughput in each cell for the network in Fig. 7.

and high-mobility cases, respectively, which is a $32 \%$ increase in throughput over the optimized traditional CAC algorithm for the same guaranteed blocking probability threshold of 0.02 . The value of $\lambda$ calculated from (15) increases from 1.65 (in the optimized traditional CAC algorithm) to 2.19 calls per unit time (in our optimization) for the no-mobility case, from 1.70 to 2.27 calls per unit time for the low-mobility case, and from 1.75 to 2.37 calls per unit time for the high-mobility case. Due to its call tradeoffs between low- and high-traffic cells, our algorithm is able to better accommodate the unequal call-arrival rates in the network and to achieve higher throughput in all the cells for the same guaranteed GoS.

Fig. 13 presents the values of the throughput for the entire network for both our optimization and the optimized traditional $\mathrm{CAC}$ algorithm as the blocking probability threshold is varied

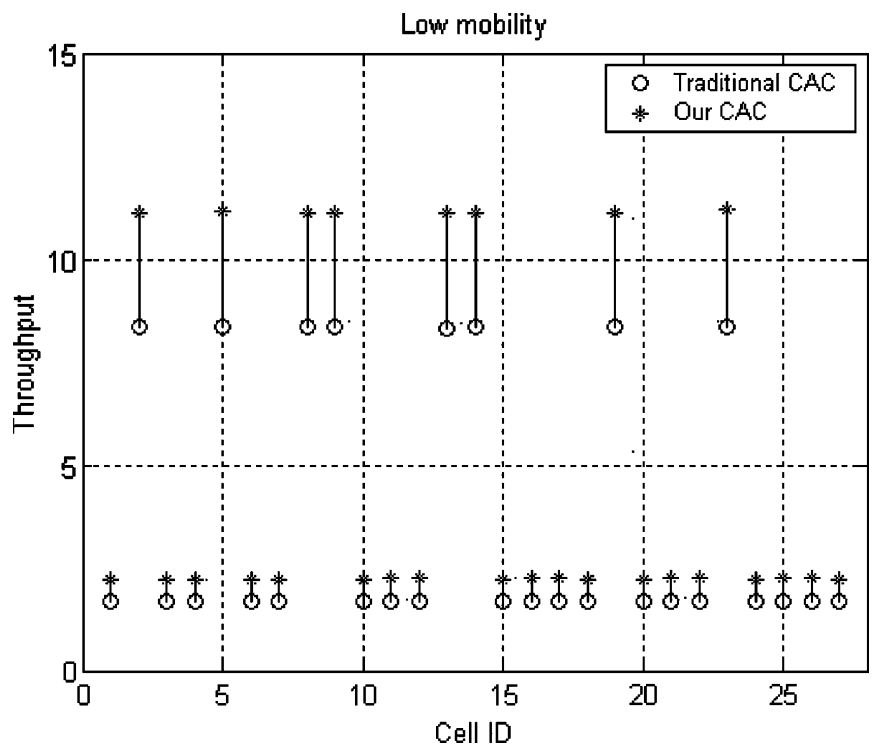

Fig. 11. Maximum throughput in each cell for the network in Fig. 8.

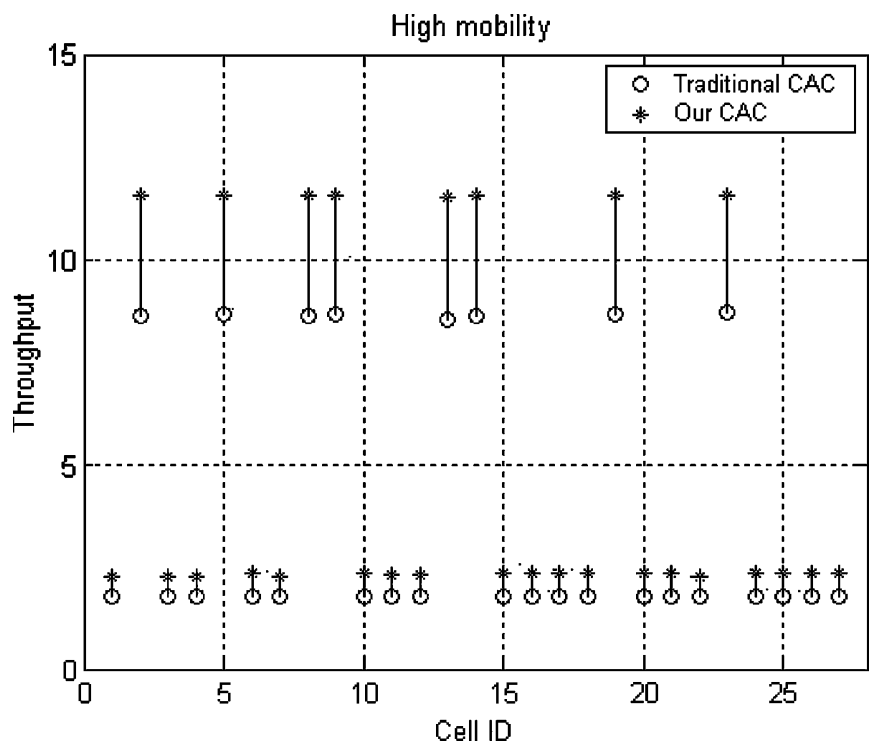

Fig. 12. Maximum throughput in each cell for the network in Fig. 9.

from 0.01 to 0.1 . Fig. 13 clearly demonstrates the significant increase in network throughput as a result of using our optimization versus the optimized traditional algorithm.

\section{Network Capacity}

In this section, we also choose $\eta=0.02$. The throughput per cell, calculated from (15), is shown in square brackets for the no-, low-, and high-mobility cases in Figs. 14-16, respectively. The network capacity for the no-, low-, and high-mobility cases is 259,265 , and 263 calls per unit time, respectively. The maximum number of calls that can be admitted in each cell is shown in parentheses in the same figures. It can be seen that for the cells on the outer edges of the coverage area, the throughput is higher. This is due to the fact that the intercell interference for these cells is smaller than that for the cells in the interior of the 


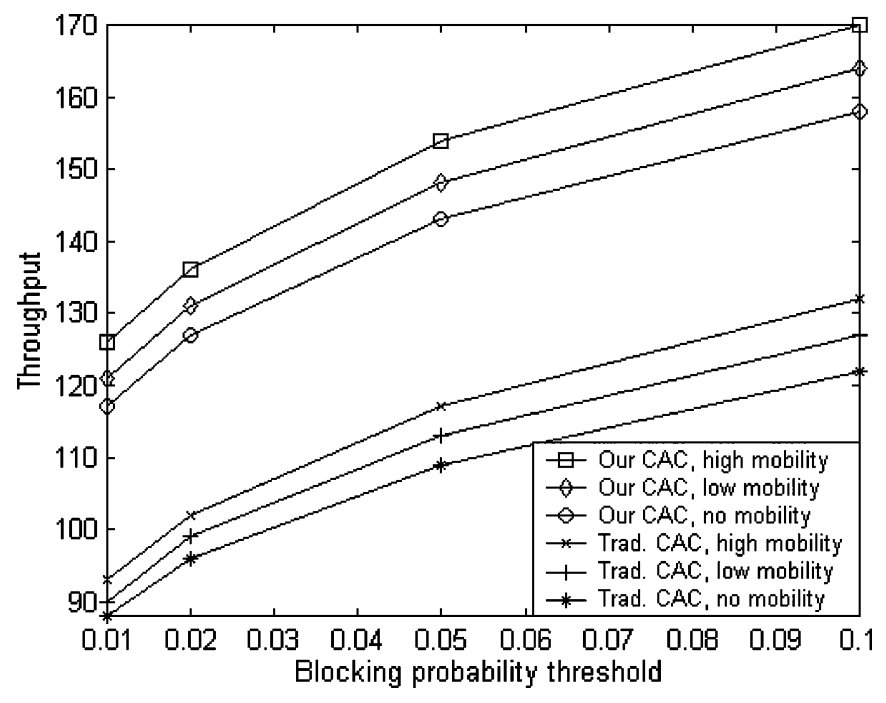

Fig. 13. Maximum throughput in the network for different values of the blocking probability threshold.

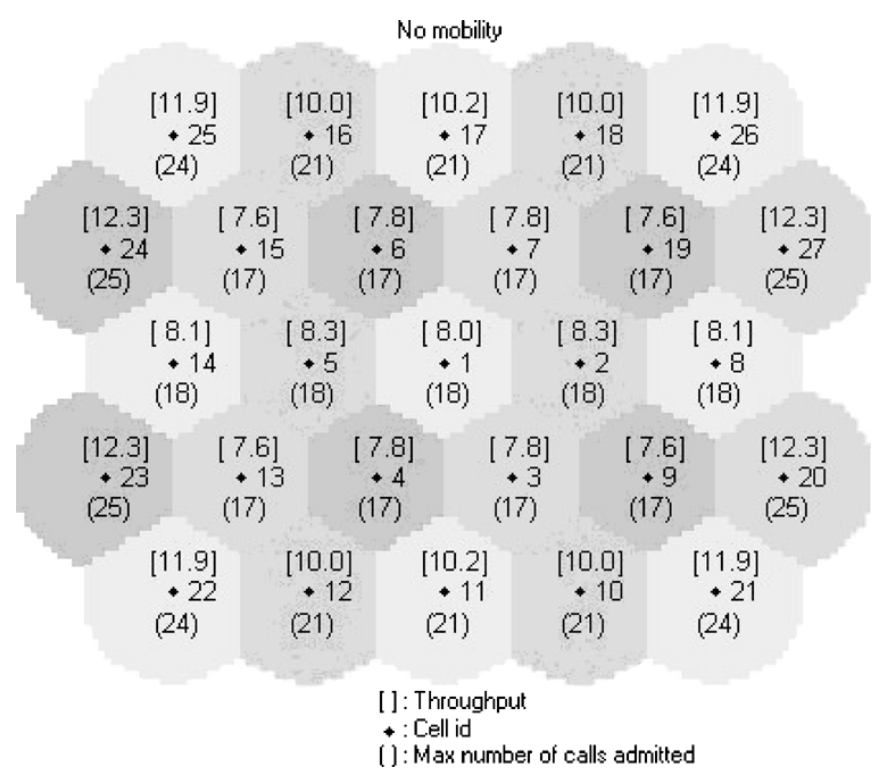

Fig. 14. Throughput and maximum number of calls allowed to be admitted per cell for the network with no mobility of users.

coverage area and, thus, the maximum number of calls that can be admitted is higher for the cells on the outer edges.

Fig. 17 presents the values of the network capacity as the blocking probability threshold is varied from 0.001 to 0.1 .

\section{CONCLUSION}

A novel approach is presented for designing a $\mathrm{CAC}$ algorithm that guarantees the QoS and achieves lower blocking probabilities for a CDMA network with arbitrary call-arrival rates. The CAC algorithm accounts for the mobility of users between cells. The design complexity is reduced substantially by reducing the set of feasible states that a CDMA network can be in to a set of admissible states when calculating the blocking probabilities. We formulated a constrained optimization problem that maximizes a revenue function that consists of two components: the

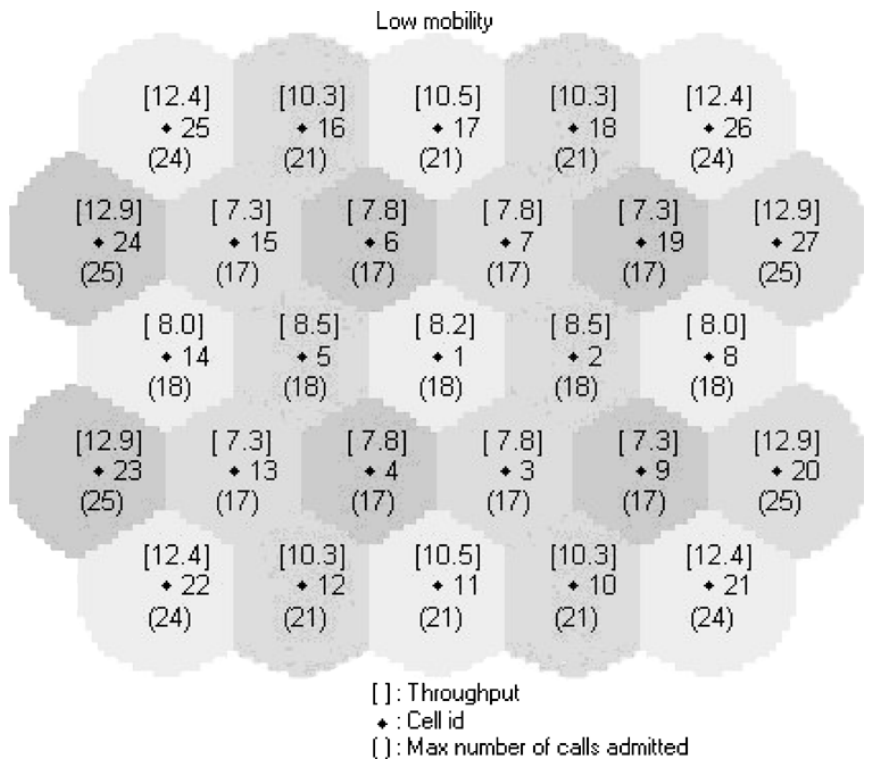

Fig. 15. Throughput and maximum number of calls allowed to be admitted per cell for the network with low mobility of users.

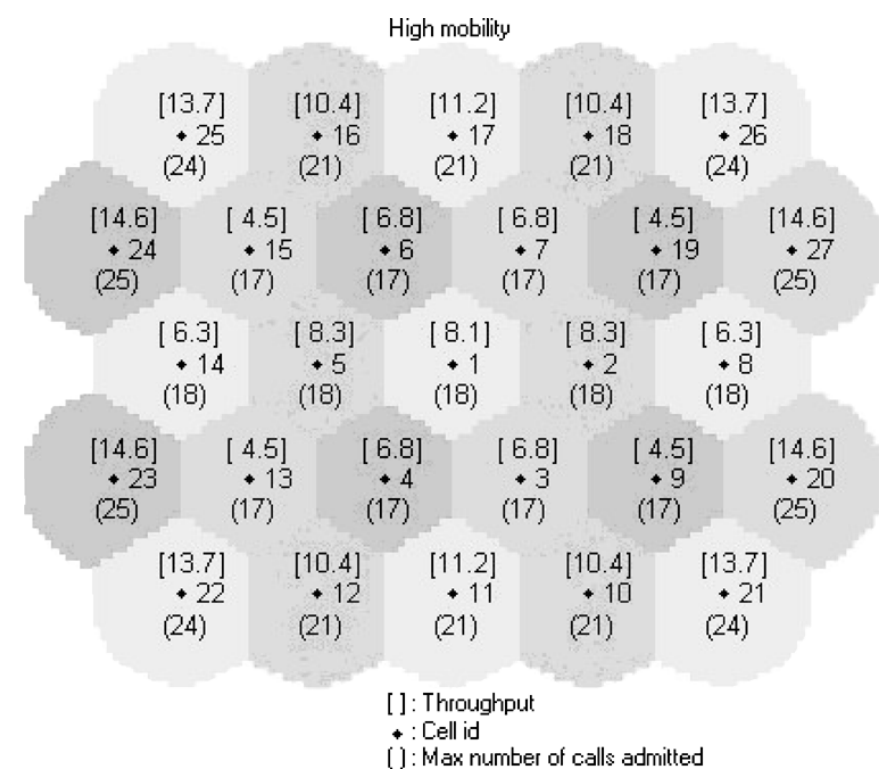

Fig. 16. Throughput and maximum number of calls allowed to be admitted per cell for the network with high mobility of users.

revenue generated by accepting in each cell a new call and the cost of a forced termination due to a handoff failure. The revenue depends implicitly on the CAC algorithm. The solution to the optimization problem is the maximum number of calls allowed to be admitted in each cell for a given blocking probability threshold and QoS requirements. For equal call-arrival rates, our CAC algorithm is identical to the optimized traditional CAC algorithm where the maximum number of calls that could be admitted in each cell is the same (ignoring edge effects). For unequal call-arrival rates, our optimization algorithm achieved a $32 \%$ increase in throughput over the optimized traditional CAC algorithm. The algorithm also calculates the network capacity, i.e., the upper bound on the throughput for a given network topology, GoS, and QoS. 


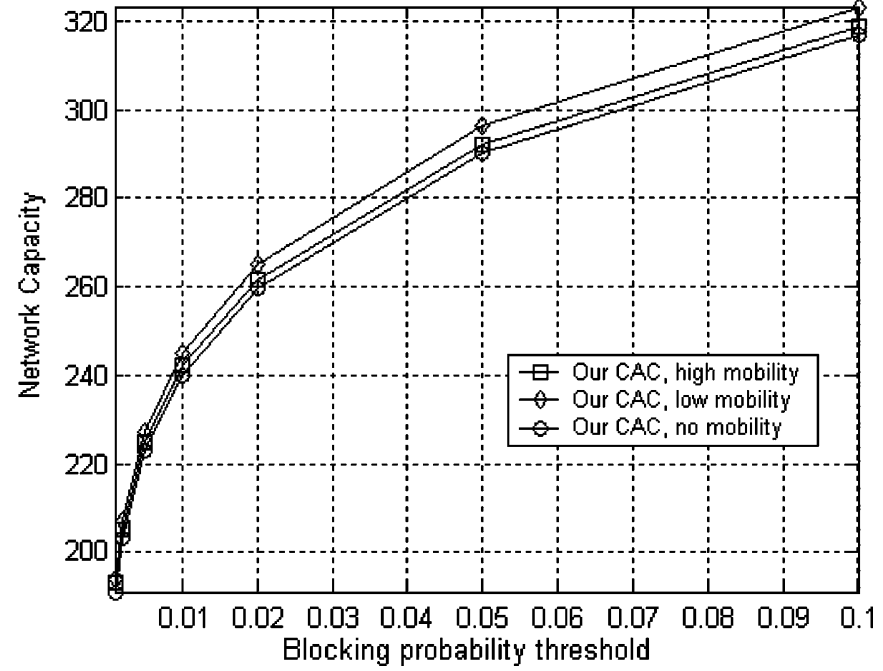

Fig. 17. Network capacity for different values of the blocking probability threshold.

\section{APPENDIX \\ DERIVATION OF THE IMPLIED COSTS}

We need to evaluate the sensitivity of the blocking probability with respect to the maximum number of calls allowed to be admitted in each cell and the offered load to aid in the solution to the optimization problems that we formulated in Section IV. To this end, we extend the function defined by (10) to one that is valid for all nonnegative $N_{i}$. In [45], the author presents the following recursive relation:

$$
B\left(A_{i}, X_{i}+1\right)=\frac{A_{i} B\left(A_{i}, X_{i}\right)}{X_{i}+1+A_{i} B\left(A_{i}, X_{i}\right)}
$$

where $X_{i}$ is not restricted to integer values. Furthermore, the author has shown that this recursive relation does not cause a systematic buildup of errors in the initial value $B\left(A_{i}, X_{i}^{(0)}\right)$. $X_{i}^{(0)}$ can be chosen to be the fractional part of $N_{i}$; that is, $X_{i}^{(0)}=N_{i}-\left\lfloor N_{i}\right\rfloor$, where $\lfloor x\rfloor$ is the largest integer no larger than $x$. Then, if $B\left(A_{i}, X_{i}^{(0)}\right)$ can be easily computed, applying (16) $\left(N_{i}-X_{i}^{(0)}\right)$ times yields $B\left(A_{i}, N_{i}\right)$. One method for computing $B\left(A_{i}, X_{i}^{(0)}\right)$ is given in [45]

$$
B\left(A_{i}, X_{i}^{(0)}\right)=C_{0}+C_{1} X_{i}^{(0)}+C_{2}\left(X_{i}^{(0)}\right)^{2}
$$

where

$$
\begin{aligned}
C_{0} & =1 \\
C_{1} & =-\frac{A_{i}+2}{\left(1+A_{i}\right)^{2}+A_{i}} \\
C_{2} & =\frac{1}{\left(1+A_{i}\right)\left[\left(1+A_{i}\right)^{2}+A_{i}\right]} .
\end{aligned}
$$

\section{A. Sensitivity Analysis with Respect to $\left(X_{i}+1\right)$}

Having formulated the equations for the calculation of the blocking probabilities, we calculate the sensitivity of the blocking probability (16) to $X_{i}+1$. The total derivative of the left-hand side of (16) with respect to the maximum number of calls admitted is given by

$$
\begin{aligned}
\frac{d B\left(A_{i}, X_{i}+1\right)}{d\left(X_{i}+1\right)}=\frac{\partial B\left(A_{i}, X_{i}+1\right)}{\partial\left(X_{i}+1\right)} & \\
& +\frac{\partial B\left(A_{i}, X_{i}+1\right)}{\partial B\left(A_{i}, X_{i}\right)} \frac{d B\left(A_{i}, X_{i}\right)}{d X_{i}} .
\end{aligned}
$$

The first partial derivative in (21) is obtained as

$$
\frac{\partial B\left(A_{i}, X_{i}+1\right)}{\partial\left(X_{i}+1\right)}=-\frac{A_{i} B\left(A_{i}, X_{i}\right)}{\left(X_{i}+1+A_{i} B\left(A_{i}, X_{i}\right)\right)^{2}}
$$

and the second partial derivative in (21) is given by

$$
\frac{\partial B\left(A_{i}, X_{i}+1\right)}{\partial B\left(A_{i}, X_{i}\right)}=\frac{\left(X_{i}+1\right) A_{i}}{\left(X_{i}+1+A_{i} B\left(A_{i}, X_{i}\right)\right)^{2}} .
$$

As for $\left(d B\left(A_{i}, X_{i}\right) / d X_{i}\right)$, it is obtained recursively using (21), with the final term calculated from (17), i.e.,

$$
\begin{aligned}
\frac{d B\left(A_{i}, X_{i}^{(0)}\right)}{d X_{i}^{(0)}} & =C_{1}+2 C_{2} X_{i}^{(0)} \\
& =-\frac{3 A_{i}+A_{i}^{2}+2-2 X_{i}^{(0)}}{\left(1+A_{i}\right)\left(1+3 A_{i}+A_{i}^{2}\right)} .
\end{aligned}
$$

\section{B. Sensitivity with Respect to $A_{i}$}

To calculate the sensitivity of the blocking probability (16) with respect to the offered load $A_{i}$, we find the total derivative of the left-hand side of (16) with respect to $A_{i}$. This is given as

$$
\begin{aligned}
\frac{d B\left(A_{i}, X_{i}+1\right)}{d A_{i}}=\frac{\partial B\left(A_{i}, X_{i}+1\right)}{\partial A_{i}} & \\
& +\frac{\partial B\left(A_{i}, X_{i}+1\right)}{\partial B\left(A_{i}, X_{i}\right)} \frac{d B\left(A_{i}, X_{i}\right)}{d A_{i}} .
\end{aligned}
$$

The first partial derivative in (25) is obtained as

$$
\frac{\partial B\left(A_{i}, X_{i}+1\right)}{\partial A_{i}}=\frac{\left(X_{i}+1\right) B\left(A_{i}, X_{i}\right)}{\left(X_{i}+1+A_{i} B\left(A_{i}, X_{i}\right)\right)^{2}}
$$

and the second partial derivative in (25) is given by (23). As for $\left(d B\left(A_{i}, X_{i}\right) / d A_{i}\right)$, it is obtained recursively using (25), with the final term calculated from (17), i.e.,

$$
\frac{d B\left(A_{i}, X_{i}^{(0)}\right)}{d A_{i}}=X_{i}^{(0)} \frac{\partial C_{1}}{\partial A_{i}}+\left(X_{i}^{(0)}\right)^{2} \frac{\partial C_{2}}{\partial A_{i}} .
$$

The partial derivatives in (27) are given by

$$
\frac{\partial C_{1}}{\partial A_{i}}=\frac{5+4 A_{i}+A_{i}^{2}}{\left(1+3 A_{i}+A_{i}^{2}\right)^{2}}
$$

and

$$
\frac{\partial C_{2}}{\partial A_{i}}=-\frac{4+8 A_{i}+3 A_{i}^{2}}{\left(1+A_{i}\right)^{2}\left(1+3 A_{i}+A_{i}^{2}\right)^{2}} .
$$




\section{Calculation of the Implied Cost with Respect to $\mathbf{N}$}

In what follows, we determine the implied costs of the revenue of the entire network with respect to the maximum number of calls admitted in a cell. The total derivative of the revenue function with respect to the maximum number of calls that are allowed to be admitted in a cell is

$$
\begin{aligned}
& \frac{d H(\mathbf{B}, \underline{\rho}, \underline{\lambda})}{d N_{k}}=\sum_{i=1}^{M}\left\{\frac{\partial H(\mathbf{B}, \underline{\rho}, \underline{\lambda})}{\partial B_{i}} \frac{d B\left(A_{i}, N_{i}\right)}{d N_{k}}\right. \\
& \left.+\frac{\partial H(\mathbf{B}, \underline{\rho}, \underline{\lambda})}{\partial \rho_{i}} \frac{d \rho\left(\mathbf{v}, \lambda_{i}, \mathcal{A}_{i}\right)}{d N_{k}}\right\} .
\end{aligned}
$$

The partial derivatives needed in (30) are obtained from (12) as

$$
\begin{aligned}
& \frac{\partial H(\mathbf{B}, \underline{\rho}, \underline{\lambda})}{\partial B_{i}}=-w_{i} \lambda_{i}-c_{i}\left(\rho_{i}-\lambda_{i}\right) \\
& \frac{\partial H(\mathbf{B}, \underline{\rho}, \underline{\lambda})}{\partial \rho_{i}}=-c_{i} B_{i} .
\end{aligned}
$$

The derivative $\left(d B\left(A_{i}, N_{i}\right) / d N_{k}\right)$ is equal to 0 if $i \neq k$. For $i=$ $k,\left(d B\left(A_{k}, N_{k}\right) / d N_{k}\right)$ was derived in Section A. The second total derivative needed in (30) can be obtained from (8) as

$$
\frac{d \rho\left(\mathbf{v}, \lambda_{i}, \mathcal{A}_{i}\right)}{d N_{k}}=\sum_{x \in \mathcal{A}_{i}} \frac{\partial \rho\left(\mathbf{v}, \lambda_{i}, \mathcal{A}_{i}\right)}{\partial \nu_{x i}} \frac{d \nu\left(B_{x}, \rho_{x}, q_{x i}\right)}{d N_{k}} .
$$

The terms $\nu\left(B_{x}, \rho_{x}, q_{x i}\right)$ are given by (7). To continue with the calculation of the implied cost, we need the partial derivatives of the offered traffic with respect to the handoff rates. From (8), we obtain $\left(\partial \rho\left(\mathbf{v}, \lambda_{i}, \mathcal{A}_{i}\right) / \partial \nu_{x i}\right)=\mathcal{I}_{\left\{x \in \mathcal{A}_{i}\right\}}$, where $\mathcal{I}_{\{D\}}$ is equal to 1 if event $D$ is true and 0 otherwise. Finally, from (7), the derivatives of the handoff rates with respect to the number of calls admitted in cell $k$ are given by

$$
\begin{aligned}
\frac{d \nu\left(B_{x}, \rho_{x}, q_{x i}\right)}{d N_{k}}=\frac{\partial \nu\left(B_{x}, \rho_{x}, q_{x i}\right)}{\partial B_{x}} \frac{d B\left(A_{x}, N_{x}\right)}{d N_{k}} & \\
& +\frac{\partial \nu\left(B_{x}, \rho_{x}, q_{x i}\right)}{\partial \rho_{x}} \frac{d \rho\left(\mathbf{v}, \lambda_{x}, \mathcal{A}_{x}\right)}{d N_{k}} .
\end{aligned}
$$

The partial derivatives needed in (34) are given by

$$
\begin{aligned}
& \frac{\partial \nu\left(B_{x}, \rho_{x}, q_{x i}\right)}{\partial B_{x}}=-q_{x i} \rho_{x} \\
& \frac{\partial \nu\left(B_{x}, \rho_{x}, q_{x i}\right)}{\partial \rho_{x}}=\left(1-B_{x}\right) q_{x i} .
\end{aligned}
$$

Equations (35) and (36) are substituted back in (34), which is substituted in (33), which results in expressions of the total derivatives $\left(d \rho\left(\mathbf{v}, \lambda_{i}, \mathcal{A}_{i}\right) / d N_{k}\right)$ in terms of $\left(d B\left(A_{x}, N_{x}\right) / d N_{k}\right)$ and $\left(d \rho\left(\mathbf{v}, \lambda_{x}, \mathcal{A}_{x}\right) / d N_{k}\right)$. Then, (21) is substituted in (33), resulting in expressions of the total derivatives $\left(d \rho\left(\mathbf{v}, \lambda_{i}, \mathcal{A}_{i}\right) / d N_{k}\right)$ in terms of $\left(d \rho\left(\mathbf{v}, \lambda_{x}, \mathcal{A}_{x}\right) / d N_{k}\right)$. This results in a set of simultaneous linear equations, which is then solved and the results substituted back in (30) along with (31) and (32). This completes the derivation of the implied cost of the revenue with respect to the maximum number of calls that are allowed to be admitted in each cell.

\section{Calculation of the Implied Cost with Respect to $\underline{\lambda}$}

In what follows, we determine the implied costs of the revenue with respect to the new-call-arrival rates. The total derivative of the revenue function with respect to the new-call-arrival rates is given by

$$
\begin{array}{r}
\frac{d H(\mathbf{B}, \underline{\rho}, \underline{\lambda})}{d \lambda_{k}}=\frac{\partial H(\mathbf{B}, \underline{\rho}, \underline{\lambda})}{\partial \lambda_{k}}+\sum_{i=1}^{M}\left\{\frac{\partial H(\mathbf{B}, \underline{\rho}, \underline{\lambda})}{\partial B_{i}} \frac{d B\left(A_{i}, N_{i}\right)}{d \lambda_{k}}\right. \\
\left.+\frac{\partial H(\mathbf{B}, \underline{\rho}, \underline{\lambda})}{\partial \rho_{i}} \frac{d \rho\left(\mathbf{v}, \lambda_{i}, \mathcal{A}_{i}\right)}{d \lambda_{k}}\right\} \cdot \quad \text { (37) }
\end{array}
$$

The first term in (37) is obtained as

$$
\frac{\partial H(\mathbf{B}, \underline{\rho}, \underline{\lambda})}{\partial \lambda_{k}}=w_{k}\left(1-B_{k}\right)+c_{k} B_{k}
$$

The remaining partial derivatives needed in (37) are given in (31) and (32). From (16) we get

$$
\frac{d B\left(A_{i}, N_{i}\right)}{d \lambda_{k}}=\frac{\partial B\left(A_{i}, N_{i}\right)}{\partial A_{i}} \frac{\partial A_{i}}{\partial \rho_{i}} \frac{d \rho\left(\mathbf{v}, \lambda_{i}, \mathcal{A}_{i}\right)}{d \lambda_{k}} .
$$

The first partial derivative in (39) was derived in Section B. The second partial derivative in (39) is given by $\left(\partial A_{i} / \partial \rho_{i}\right)=$ $\left(1 / \mu_{i}\right)$. The total derivative needed in (39) and (37) can be obtained from (8) as

$$
\begin{aligned}
\frac{d \rho\left(\mathbf{v}, \lambda_{i}, \mathcal{A}_{i}\right)}{d \lambda_{k}}=\frac{\partial \rho\left(\mathbf{v}, \lambda_{i}, \mathcal{A}_{i}\right)}{\partial \lambda_{k}} & \\
& +\sum_{x \in \mathcal{A}_{i}} \frac{\partial \rho\left(\mathbf{v}, \lambda_{i}, \mathcal{A}_{i}\right)}{\partial \nu_{x i}} \frac{d \nu_{x i}\left(B_{x}, \rho_{x}\right)}{d \lambda_{k}}
\end{aligned}
$$

where $\left(\partial \rho\left(\mathbf{v}, \lambda_{i}, \mathcal{A}_{i}\right) / \partial \lambda_{k}\right)=\mathcal{I}_{\{i=k\}}$. From (8) we get $\left(\partial \rho\left(\mathbf{v}, \lambda_{i}, \mathcal{A}_{i}\right) / \partial \nu_{x i}\right)=\mathcal{I}_{\left\{x \in \mathcal{A}_{i}\right\}}$. Finally, from (7), the derivatives of the handoff rates with respect to the call-arrival rate are given by

$$
\begin{aligned}
& \frac{d \nu\left(B_{x}, \rho_{x}, q_{x i}\right)}{d \lambda_{k}}=\frac{\partial \nu\left(B_{x}, \rho_{x}, q_{x i}\right)}{\partial B_{x}} \frac{d B\left(A_{x}, N_{x}\right)}{d \lambda_{k}} \\
&+\frac{\partial \nu_{x i}\left(B_{x}, \rho_{x}\right)}{\partial \rho_{x}} \frac{d \rho\left(\mathbf{v}, \lambda_{x}, \mathcal{A}_{x}\right)}{d \lambda_{k}}
\end{aligned}
$$

The partial derivatives needed in (41) were obtained in (35) and (36), which are substituted back in (41), which is substituted in (40), resulting in expressions of the total derivatives $\left(d \rho\left(\mathbf{v}, \lambda_{i}, \mathcal{A}_{i}\right) / d \lambda_{k}\right)$ in terms of $\left(d B\left(A_{x}, N_{x}\right) / d \lambda_{k}\right)$ and $\left(d \rho\left(\mathbf{v}, \lambda_{x}, \mathcal{A}_{x}\right) / d \lambda_{k}\right)$. Then (39) is substituted in (40), which results in expressions of the total derivatives $\left(d \rho\left(\mathbf{v}, \lambda_{i}, \mathcal{A}_{i}\right) / d \lambda_{k}\right)$ in terms of $\left(d \rho\left(\mathbf{v}, \lambda_{x}, \mathcal{A}_{x}\right) / d \lambda_{k}\right)$. This results in a set of simultaneous linear equations, which is then solved and the results substituted back in (37) along with (38), (31), and (32). This completes the derivation of the implied cost and the values of the derivatives of the revenue with respect to the call-arrival rates. Implied costs capture the effect of increases in the call-arrival rate in one cell on the throughput of the entire network. 


\section{REFERENCES}

[1] A. Viterbi and A. J. Viterbi, "Erlang capacity of a power controlled CDMA system," IEEE J. Sel. Areas Commun., vol. 11, no. 6, pp. 892-900, Aug. 1993.

[2] J. Evans and D. Everitt, "On the teletraffic capacity of CDMA cellular networks," IEEE Trans. Veh. Technol., vol. 48, no. 1, pp. 153-165, Jan. 1999.

[3] A. Fapojuwo, "Radio capacity of direct sequence code division multiple access mobile radio systems," Inst. Elect. Eng. Proc.-I, vol. 140, no. 5, pp. 402-408, 1993.

[4] M. Frullone, G. Riva, P. Grazioso, and M. Missiroli, "Comparisons of multiple access schemes for personal communication systems in a mixed cellular environment," IEEE Trans. Veh. Technol., vol. 43, no. 1, pp. 99-109, Feb. 1994

[5] Z. Liu and M. E. Zarki, "SIR-based call admission control for DS-CDMA cellular systems," IEEE Trans. Commun., vol. 12, no. 4, pp. 638-644, May 1994.

[6] J. Wu and B. Hwang, "Call admission control and scheduling algorithm for a CDMA system," in IEEE Pacific Rim Conf. Communications, Computers and Signal Processing, vol. 1, 2001, pp. 27-30.

[7] Y. Ishikawa and N. Umeda, "Capacity design and performance of call admission control in cellular CDMA systems," IEEE J. Sel. Areas Commun., vol. 15, no. 8, pp. 1627-1635, Oct. 1997.

[8] I. Kim, B. Shin, and D. Lee, "SIR-based call admission control by intercell interference prediction for DS-CDMA systems," IEEE Commun. Lett., vol. 4, no. 1, pp. 29-31, Jan. 2000

[9] W. Ying, Z. Jingmei, W. Weidong, and Z. Ping, "Call admission control in hierarchical cell structure," in Proc. IEEE Veh. Technol. Conf., vol. 4, May 2002, pp. 1955-1959.

[10] D. Kim, "On upper bounds of SIR-based call admission threshold in power-controlled DS-CDMA mobile systems," IEEE Commun. Lett., vol. 6 , no. 1, pp. 13-15, Jan. 2002.

[11] T. Tugcu and C. Ersoy, "A novel call admission scheme based on interference for DS-CDMA systems," in Proc. IEEE Symp. Communications and Vehicular Technology, 2000, pp. 109-114.

[12] I. Koo, S. Bahng, and K. Kim, "Resource reservation in call admission control schemes for CDMA systems with nonuniform traffic distribution among cells," in Proc. IEEE Veh. Technol. Conf., vol. 1, 2003, pp. $438-441$.

[13] X. Li, H. Xiaodong, and W. Jianfang, "Research and simulation of intelligent call admission control method," in Proc. IEEE Region 10 Conf. Computers, Communications, Control and Power Engineering, vol. 2, Oct. 2002, pp. 763-766.

[14] D. Everitt, "Analytic traffic models of CDMA cellular networks," in Proc. 14th Int. Teletraffic Congress, Jun. 1994, pp. 349-356.

[15] G. Foschini, B. Gopinath, and Z. Miljanic, "Channel cost of mobility," IEEE Trans. Veh. Technol., vol. 42, no. 4, pp. 414-424, Nov. 1993.

[16] D. Lam, D. Cox, and J. Widom, "Traffic modeling for personal communication services," IEEE Commun. Mag., no. 2, pp. 79-87, Feb. 1997.

[17] J. Markoulidakis, G. Lyberopoulos, D. Tsirkas, and E. Sykas, "Mobility modeling in third-generation mobile telecommunications systems," IEEE Pers. Commun. Mag., pp. 41-56, Aug. 1997.

[18] K. Leung, W. Massey, and W. Whitt, "Traffic models for wireless communication networks," IEEE J. Sel. Areas Commun., vol. 12, no. 8, pp. 1353-1364, Oct. 1994

[19] K. Nakano, K. Saita, N. Karasawa, M. Sengoku, and S. Shinoda, "Teletraffic characteristics in cellular systems and traffic flow in road systems," in Proc. IEEE Veh. Technol. Conf., vol. 2, 1999, pp. 1193-1197.

[20] P. Orlik and S. Rappaport, "A model for teletraffic performance and channel holding time characterization in wireless cellular communication with general session and dwell time distributions," IEEE J. Sel. Areas Commun., vol. 16, no. 5, pp. 788-803, Jun. 1998.

[21] P. Wirth, "The role of teletraffic modeling in the new communications paradigms," IEEE Commun. Mag., vol. 35, no. 8, pp. 86-92, Aug. 1997.

[22] M. Zonoozi and P. Dassanayake, "User mobility modeling and characterization of mobility patterns," IEEE J. Sel. Areas Commun., vol. 15, no. 7, pp. 1239-1252, Sep. 1997.

[23] T. Tugcu and C. Ersoy, "Application of a realistic mobility model to call admissions in DS-CDMA cellular systems," in Proc. IEEE Veh. Technol. Conf., vol. 2, 2001, pp. 1047-1051.

[24] L. Badia, M. Zorzi, and A. Gazzini, "On the impact of user mobility on call admission control in WCDMA systems," in Proc. IEEE Veh. Technol. Conf., vol. 1, Sep. 2002, pp. 121-126.
[25] R. Akl, M. Hegde, M. Naraghi-Pour, and P. Min, "Multi-cell CDMA network design," IEEE Trans. Veh. Technol., vol. 50, no. 3, pp. 711-722, May 2001.

[26] C. Vargas, M. Hegde, and M. Naraghi-Pour, "Implied costs for multi-rate wireless networks," J.Wireless Networks, vol. 10, no. 3, pp. 323-337, May 2004.

[27] V. Istratescu, Fixed Point Theory: An Introduction. Amsterdam, The Netherlands: D. Reidel, 1981

[28] D. Mitra, J. Morrison, and K. Ramakrishnan, "ATM network design and optimization: A multirate loss network framework," IEEE/ACM Trans. Networking, vol. 4, no. 4, pp. 531-543, Aug. 1996.

[29] C. Vargas-Rosales, "Communication network design and evaluation using shadow prices," Ph.D. dissertation, Louisiana State Univ., Baton Rouge, 1996.

[30] F. Kelly, "Routing in circuit-switched network: Optimization, shadow prices and decentralization," Adv. Appl. Prob., vol. 20, pp. 112-144, 1988.

[31] C. Vargas, M. Hegde, and M. Naraghi-Pour, "Implied costs in wireless networks," in Proc. IEEE Veh. Technol. Conf., vol. 2, May 1998, pp. 904-908.

[32] _ - "Blocking effects of mobility and reservations in wireless networks," in Proc. IEEE Int. Conf. Communications, vol. 3, Jun. 1998, pp. 1612-1616.

[33] R. Akl, M. Hegde, and P. Min, "Effects of call arrival rate and mobility on network throughput in multi-cell CDMA," in IEEE Int. Conf. Communications, vol. 3, Jun. 1999, pp. 1763-1767.

[34] F. Kelly, "Blocking probabilities in large circuit switched networks," Adv. Appl. Prob., vol. 18, pp. 473-505, 1986.

[35] G. Ash, R. Cardwell, and R. Murray, "Design and optimization of networks with dynamic routing," Bell Syst. Tech. J., vol. 60, pp. 1787-1820, 1981.

[36] G. Nemhauser and L. Wolsey, Integer and Combinatorial Optimization. New York: Wiley, 1988.

[37] M. Powell, "A fast algorithm for nonlinearly constrained optimization calculations," Numer. Anal., vol. 630, pp. 144-157, 1978.

[38] T. Coleman and Y. Li, "An interior, trust region approach for nonlinear minimization subject to bounds," SIAM J. Optimizat., vol. 6, pp. 418-445, 1996

[39] S. Han, "A globally convergent method for nonlinear programming," $J$. Optimizat. Theory Applicat., vol. 22, no. 3, pp. 297-309, 1977.

[40] P. Gill, W. Murray, and M. Wright, Practical Optimization. New York: Academic, 1981.

[41] S. Kirkpatrick, C. Gelatt, and M. Vecchi, "Optimization by simulated annealing," Sci., vol. 220, no. 4593, pp. 671-680, 1983.

[42] L. Ingber, "Simulated annealing: Practice versus theory," J. Math. Computat. Modeling, vol. 18, no. 11, pp. 29-57, 1993.

[43] T. Rappaport, Wireless Communications Principles and Practice. Englewood Cliffs, NJ: Prentice-Hall, 1996.

[44] A. Viterbi, CDMA Principles of Spread Spectrum Communication. Reading, MA: Addison-Wesley, 1995.

[45] Y. Rapp, "Planning of junction network in a multi-exchange area," Ericson Technics, vol. 20, no. 1, pp. 77-130, 1964.

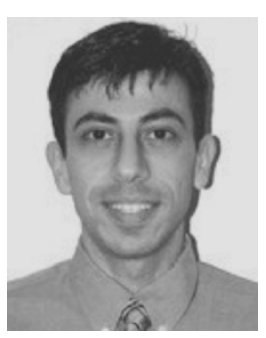

Robert G. Akl (S'94-M'00) received the B.S. degree in computer science from Washington University, St. Louis, MO, in 1994 and the B.S., M.S. and D.Sc. degrees in electrical engineering in 1994, 1996, and 2000 , respectively.

From October 2000 to December 2001, he was a Senior Systems Engineer with the Comspace Corporation, Coppell, TX. In 2002, he was an Assistant Professor in the Department of Electrical and Computer Engineering, University of New Orleans, New Orleans, LA. He currently is an Assistant Professor in the Department of Computer Science and Engineering, University of North Texas, Denton. His research interests include wireless communication and network design and optimization.

Dr. Akl received the Dual Degree Engineering Outstanding Senior Award from Washington University in 1993. 


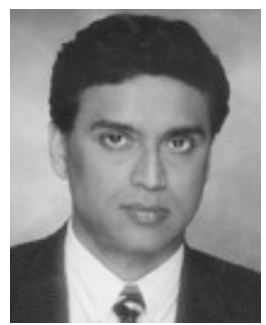

Manju V. Hegde (S'84-M'87) received the B.Tech. degree in electrical engineering from the Indian Institute of Technology, Bombay, India, and the Ph.D. degree in computer, information, and control engineering from the University of Michigan, Ann Arbor, in 1987.

From 1987 to 1997, he was an Assistant Professor and then an Associate Professor in the Electrical and Computer Engineering Department, Louisiana State University, Baton Rouge. From 1997 to 2003, he was an Associate Professor of Electrical Engineering at Washington University, St. Louis, MO. From 1997 to 1999, he was Chief Scientist with MinMax Technologies, St. Louis, MO, a fabless semiconductor manufacturer. He is a Founder of Celox Networks, St. Louis, MO, an edge router manufacturer, and served as its CTO from 1999 to 2002. He is a Founder of Ageia Technologies, St. Louis, MO, a fabless semiconductor manufacturer, which he co-founded in 2002 and currently is Chief Executive Officer. He has been an Editor for the Journal of Network and Systems Management from 1997 to 2002.

His research interests include communication and information theory, switch fabric architectures, coding theory, and telecommunication networks.

Dr. Hegde received the Outstanding Graduate Student Award in Computer Information and Control Engineering from the University of Michigan.

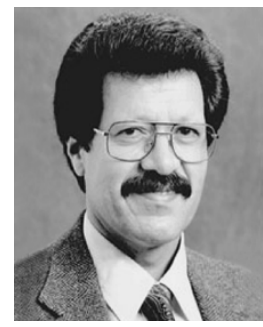

Mort Naraghi-Pour (S'81-M'87) was born in Tehran, Iran, on May 15, 1954. He received the B.S.E. degree from Tehran University in 1977 and the M.S. and Ph.D. degrees in electrical engineering from the University of Michigan, Ann Arbor, in 1983 and 1987, respectively. In 1978, he was a student at the Philips International Institute, Eindhoven, The Netherlands, where he also did research with the telecommunication switching group of the Philips Research Laboratories.

Since August 1987, he has been with the Department of Electrical and Computer Engineering, Louisiana State University, Baton Rouge, where he currently is an Associate Professor. From June 2000 to January 2002, he was a Senior Member of Technical Staff at Celox Networks, Inc., St. Louis, MO, a network equipment manufacturer. His research and teaching interests are in wireless communications and high speed networks.

Dr. Naraghi-Pour has served as a Session Organizer, Session Chair, and Member of the Technical Program Committee for many international conferences. 